\title{
A note on lattices with group actions
}

\author{
Tsuyoshi Atsumi
}

(Received October 30, 1996)

\begin{abstract}
By using a lattice version of Hayden's result we generalize a Jacobi's formula for the theta series of the dual lattice when a finite group acts on the lattice. This solves a problem posed by Yoshida.
\end{abstract}

Key words: lattice, group, dual lattice, Jacobi's formula.

\section{Introduction}

In his paper [6] Yoshida proved the following result.

Result There is a generalization of MacWilliams identity [4] to codes with group actions.

Moreover he raised the following problem in [6].

Problem What can we say about lattices with group actions? Can we define the equivariant version of theta functions?

We solve the problem above. In this paper we shall prove that there is a lattice version of his result.

We introduce notation and terminology in lattice theory. Let $V$ be the real $n$-dimensional space $\mathbf{R}^{n}$. A lattice $\Lambda$ [5] is a subgroup of $V$ satisfying one of the following equivalent conditions:

i) $\Lambda$ is discrete and $V / \Lambda$ is compact;

ii) $\Lambda$ is discrete and generates the $\mathbf{R}$-vector space $V$;

iii) There exists an $\mathbf{R}$-basis $\left(e_{1}, \ldots, e_{n}\right)$ of $V$ which is a $\mathbf{Z}$-basis of $\Lambda$ (i.e. $\left.\Lambda=\mathbf{Z} e_{1} \oplus \cdots \oplus \mathbf{Z} e_{n}\right)$.

Let

$$
\begin{gathered}
e_{1}=\left(e_{11}, \ldots, e_{1 n}\right), \\
e_{2}=\left(e_{21}, \ldots, e_{2 n}\right), \\
\vdots \\
e_{n}=\left(e_{n 1}, \ldots, e_{n n}\right)
\end{gathered}
$$


be the coordinates of the basis vectors of $\Lambda$ given in (iii). The $n \times n$ matrix $M$ with $(i, j)$-entry equal to $e_{i j}$ is called a generator matrix for $\Lambda$. The determinant of $\Lambda$ is defined to be $\operatorname{det} \Lambda=|\operatorname{det} M|$.

Given two vectors $\mathbf{u}=\left(u_{1}, \ldots, u_{n}\right), \mathbf{v}=\left(v_{1}, \ldots, v_{n}\right)$ of $V$, their inner product will be denoted by $\mathbf{u} \cdot \mathbf{u}$ or $(\mathbf{u}, \mathbf{u})$. The dual lattice $\Lambda^{\perp}$ of $\Lambda$ is defined by

$$
\Lambda^{\perp}=\left\{\mathbf{u} \in \mathbf{R}^{n} \mid \mathbf{u} \cdot \mathbf{v}=u_{1} v_{1}+\cdots+u_{n} v_{n} \in \mathbf{Z} \text { for all } \mathbf{v} \in \Lambda\right\} .
$$

The theta series $\Theta_{\Lambda}(z)$ of a lattice $\Lambda$ is given by

$$
\Theta_{\Lambda}(z)=\sum_{\mathbf{u} \in \Lambda} q^{\mathbf{u} \cdot \mathbf{u}}
$$

where $q=e^{\pi i z}$. Jacobi's formula for the theta series of the dual lattice:

$$
\Theta_{\Lambda^{\perp}}(z)=(\operatorname{det} \Lambda)(i / z)^{n / 2} \Theta_{\Lambda}(-1 / z) .
$$

In section 2 we shall define $G$-lattices and give our theorem on them, which is a generalization of (1).

In section 3 we shall prove Lemma 1 which does not seem to be trivial and our theorem by using a lattice version of Hayden' theorem.

For notation and terminology, see [2] and [5] for lattice theory, [6] for lattices with group actions.

In particular, $G$ is a finite group, $\mathbf{R} G$ is a group ring over $\mathbf{R}$.

\section{Lattices with group actions}

From now on we assume that $G$ is a finite permutation group on the coordinates of $V$. Then we can define a natural action of $G$ on $V$ as follows: If $\mathbf{v}=\left(v_{1}, \ldots, v_{n}\right) \in V$ and $g \in G$, we let $\mathbf{v} g=\left(x_{1}, \ldots, x_{n}\right)$ where for $i=1, \ldots, n, x_{i}=v_{i g^{-1}}$. In this way $V$ becomes an $\mathbf{R} G$-module and every element of $\mathbf{R} G$ is a linear, orthogonal transformation of $V$. A $G$-lattice is a lattice which is also a $\mathbf{Z} G$-submodule of $V$.

As in [1], the operator $\theta$ is defined by

$$
\theta=\frac{1}{|G|} \sum_{g \in G} g \text {. }
$$

Here we note that $\theta \in \mathbf{R} G, V \theta=\{\mathbf{v} \in V \mid \mathbf{v} g=\mathbf{v}$ for all $g \in G\}, \theta^{T}=\theta$ and $\theta^{2}=\theta$, where $\theta^{T}$ is the transpose of $\theta$ (see [1]). Let $C_{1}, \ldots, C_{t}$ be the orbits of the coordinates of $V$ under the action of $G$. Let $m_{i}$ be the orbit 
length of $C_{i}$. Define $\bar{C}_{i}$ as the vector of $V$ which has $1 / \sqrt{m_{i}}$ as its entry for every point of $C_{i}$ and 0 elsewhere. (This definition of the $\bar{C}_{i}$ 's is similar to that in the proof of Theorem 4.3 in [1]). Then each of $\bar{C}_{1}, \ldots, \bar{C}_{t}$ is in $V \theta$ and every element $\mathbf{u}$ of $V \theta$ is of the form

$$
\mathbf{u}=\sum_{i=1}^{t} x_{i} \bar{C}_{i}
$$

The vector space $V \theta$ is of dimension $t$.

For vectors $\mathbf{a}, \mathbf{b}$ of $V \theta$, the inner product $\mathbf{a} \circ \mathbf{b}$ of $\mathbf{a}$ and $\mathbf{b}$ is defined by

$$
\mathbf{a} \circ \mathbf{b}=a_{1} b_{1}+\cdots+a_{t} b_{t},
$$

where $\mathbf{a}=\sum_{i=1}^{t} a_{i} \bar{C}_{i}$ and $\mathbf{b}=\sum_{i=1}^{t} b_{i} \bar{C}_{i}$.

Let $D$ be a lattice in $V \theta$. (That is, there exists an $\mathbf{R}$-basis consisting of $t$ elements of $V \theta$ which is a $\mathbf{Z}$-basis of $D$.) $D_{G}^{\perp}$ is the dual of $D$ in $V \theta$ with respect to the inner product (2). The norm of $\mathbf{u} \in D$ is $\mathbf{u} \circ \mathbf{u}$. We describe the theta series $\Theta_{D}(z)$ of a sublattice $D$ in $V \theta$ as follows:

$$
\Theta_{D}(z)=\sum_{\mathbf{u} \in D} q^{\mathbf{u} \circ \mathbf{u}}
$$

where $q=e^{\pi i z}$. Then we have the following:

Theorem Let $\Lambda$ be a $G$-lattice and let $\Lambda_{0}=\{\mathbf{r} \in \Lambda \mid \mathbf{r} \theta \in \Lambda\}$. Then the following holds:

(i) $\Lambda_{0} \theta$ is a lattice.

(ii) $\Theta_{\Lambda_{0}^{\perp} \theta}(z)=\left(\operatorname{det} \Lambda_{0} \theta\right)(i / z)^{t / 2} \Theta_{\Lambda_{0} \theta}(-1 / z)$.

If $G$ is trivial, that is, $G=\{e\}$, the equation above reduces to (1). Note that $\Lambda_{0} \theta=\Lambda \cap \Lambda \theta=\{\mathbf{v} \in \Lambda \mid \mathbf{v} g=\mathbf{v}$ for all $g \in G\}$.

\section{Proof of theorem}

We prove the following lemma which is part (i) of our theorem.

Lemma 1 Let $\Lambda$ be a G-lattice and let $\Lambda_{0}=\{\mathbf{r} \in \Lambda \mid \mathbf{r} \theta \in \Lambda\}$. Then $\Lambda_{0} \theta$ is a lattice.

Proof. First we shall show that $|G|(\Lambda \cap \Lambda \theta)\left(=|G| \Lambda_{0} \theta\right)$ is a lattice. Let $\left(e_{1}, \ldots, e_{n}\right)$ be a $\mathbf{Z}$-basis of $\Lambda$ which is also an $\mathbf{R}$-basis of $V$. Since $\left(e_{1}, \ldots, e_{n}\right)$ is an $\mathbf{R}$-basis of $V$, we see that vectors $|G| e_{1} \theta, \ldots,|G| e_{n} \theta$ of 
$|G|(\Lambda \cap \Lambda \theta)$ generate $V \theta$. This shows that

$$
V \theta \subseteq R\left(|G| e_{1} \theta\right)+\cdots+R\left(|G| e_{n} \theta\right) .
$$

On the other hand, clearly $|G|(\Lambda \cap \Lambda \theta) \subseteq V \theta$, hence we have

$$
V \theta=R\left(|G| e_{1} \theta\right)+\cdots+R\left(|G| e_{n} \theta\right) .
$$

$\Lambda$ is a free $\mathbf{Z}$-module and $|G|(\Lambda \cap \Lambda \theta)$ is a $\mathbf{Z}$-submodule of $\Lambda$. So there exists a basis $\left(m_{1}, \ldots, m_{n}\right)$ for $\Lambda$, and non-zero elements $\delta_{1}, \ldots, \delta_{r}, r \leq n$, in $\mathbf{Z}$ such that $\delta_{i} \mid \delta_{i+1} 1 \leq i \leq r-1$, and such that vectors $\delta_{1} m_{1}, \ldots, \delta_{r} m_{r}$ forms a basis for $|G|(\Lambda \cap \Lambda \theta)$ (see [3, pp 97]). Since $\left(m_{1}, \ldots, m_{n}\right)$ is an $\mathbf{R}$-basis of $V$, vectors $\delta_{1} m_{1}, \ldots, \delta_{r} m_{r}$ are linearly independent over $\mathbf{R}$. From this and (3) it follows that

$$
V \theta=\mathbf{R}\left(\delta_{1} m_{1}\right) \oplus \cdots \oplus \mathbf{R}\left(\delta_{r} m_{r}\right),
$$

which shows that $r=t$. This proves that $|G|(\Lambda \cap \Lambda \theta)$ is a lattice. So is $\Lambda \cap \Lambda \theta$.

In order to prove Theorem we need the following proposition which is a lattice version of Hayden's theorem [1].

Proposition 1 Under the same notation as in Lemma 1, we have the following:

$$
\left(\Lambda_{0} \theta\right)^{\perp}=\operatorname{Ker} \theta \oplus \Lambda_{0}^{\perp} \theta
$$

Proof. Our proof is similar to the proof of Theorem 4.2 in [1]. We note that $\Lambda_{0}$ is a $\mathbf{Z} G$-submodule of $G$-lattice $\Lambda, \theta^{T}=\theta$ and $\theta^{2}=\theta$. If $\mathbf{r} \in \Lambda_{0}$, $\hat{\mathbf{r}} \in \Lambda_{0}^{\perp}$ and $\mathbf{y} \in \operatorname{Ker} \theta^{T}$, we have

$$
\left(\hat{\mathbf{r}} \theta^{T}, \mathbf{r} \theta\right)=\left(\hat{\mathbf{r}}, \mathbf{r} \theta^{2}\right)=(\hat{\mathbf{r}}, \mathbf{r} \theta) \in Z
$$

because $\mathbf{r} \theta \in \Lambda \cap \Lambda \theta \subseteq \Lambda_{0}$ and

$$
(\mathbf{y}, \mathbf{r} \theta)=\left(\mathbf{y} \theta^{T}, \mathbf{r}\right)=0 \in Z
$$

This shows that

$$
\operatorname{Ker} \theta+\Lambda_{0}^{\perp} \theta \subseteq\left(\Lambda_{0} \theta\right)^{\perp} .
$$

If $\mathbf{r} \in \Lambda_{0}, \mathbf{y} \in\left(\Lambda_{0} \theta\right)^{\perp}$, we have

$$
\left(\mathbf{y} \theta^{T}, \mathbf{r}\right)=(\mathbf{y}, \mathbf{r} \theta) \in Z \text {. }
$$


So

$$
\mathbf{y} \theta^{T}=\mathbf{y} \theta \in \Lambda_{0}^{\perp} .
$$

Hence

$$
\mathbf{y}=\mathbf{y}-\mathbf{y} \theta+(\mathbf{y} \theta) \theta \in \operatorname{Ker} \theta+\Lambda_{0}^{\perp} \theta .
$$

This implies that

$$
\left(\Lambda_{0} \theta\right)^{\perp} \subseteq \operatorname{Ker} \theta+\Lambda_{0}^{\perp} \theta .
$$

(4) and (5) complete the proof of Proposition 1.

We start to prove Theorem. If $\mathbf{x}=\sum_{i} x_{i} \bar{C}_{i} \in \Lambda_{0} \theta$ and $\mathbf{y}=\sum_{i} y_{i} \bar{C}_{i} \in$ $\Lambda_{0}^{\perp} \theta$, by Proposition 1 we have

$$
\mathbf{x} \circ \mathbf{y}=(\mathbf{x}, \mathbf{y}) \in Z \text {. }
$$

So

$$
\Lambda_{0}^{\perp} \theta \subseteq\left(\Lambda_{0} \theta\right)_{G}^{\perp} .
$$

Now take $\mathbf{x}=\sum_{i} x_{i} \bar{C}_{i} \in\left(\Lambda_{0} \theta\right)_{G}^{\frac{1}{G}}, \mathbf{y}=\sum_{i} y_{i} \bar{C}_{i} \in \Lambda_{0} \theta$. and observe

$$
(\mathbf{x}, \mathbf{y})=\mathbf{x} \circ \mathbf{y} \in Z \text {. }
$$

This shows that

$$
\mathbf{x} \in\left(\Lambda_{0} \theta\right)^{\perp} .
$$

Since $\mathbf{x} \in V \theta,(7)$ and Proposition 1 imply that $\mathbf{x} \in \Lambda_{0}^{\perp} \theta$.

Now we proved that

$$
\left(\Lambda_{0} \theta\right)_{G}^{\perp} \subseteq \Lambda_{0}^{\perp} \theta .
$$

From (6) and (8) it follows that

$$
\left(\Lambda_{0} \theta\right)_{G}^{\perp}=\Lambda_{0}^{\perp} \theta .
$$

Now we shall finish the proof of Theorem. Lemma 1 tells us that $\Lambda_{0} \theta$ is a lattice. Hence, we have Jacobi's formula for the theta series of the dual lattice $\left(\Lambda_{0} \theta\right)_{G}^{\perp}$ in $V \theta$ :

$$
\Theta_{\left(\Lambda_{0} \theta\right)_{G}^{\perp}}(z)=\left(\operatorname{det} \Lambda_{0} \theta\right)(i / z)^{t / 2} \Theta_{\Lambda_{0} \theta}(-1 / z) .
$$

Hence equation (9) establishes our Theorem. 
Remark. It is easy to prove that

$$
\begin{aligned}
\Lambda / \Lambda_{0} & \cong \Lambda \theta / \Lambda \cap \Lambda \theta, \\
\Lambda_{0} & =(\Lambda \cap \operatorname{Ker} \theta) \oplus(\Lambda \cap \Lambda \theta) .
\end{aligned}
$$

\section{References}

[1] Bridges W.G., Hall M. and Hayden J.L., Codes and Designs. J. Combin. Theory Ser. A 31 (1981), 155-174.

[2] Conway J.H. and Sloane N.J.A., Sphere Packings, Lattices and Groups. SpringerVerlag, New York-Berlin-Heidelberg-London-Paris-Tokyo, 1988.

[3] Curtis C.W. and Reiner I., Representation Theory of Finite Groups and Associative Algebras. Interscience, New York-London-Sydney, 1966.

[4] MacWilliams F.J. and Sloane N.J.A., The Theory of The Error-Correcting Codes. North Holland, Amsterdam-New York-Oxford, 1977.

[5] Serre J.P., Cours d'Arthmétique. Presses Universitaires de France, Paris, (1970); English translation, Springer-Verlag, New York-Berlin-Heidelberg-London-ParisTokyo, 1973.

[6] Yoshida T., MacWilliams Identities for Linear Codes with Group Action. Kumamoto J. Math. 6 (1993), 29-45.

Department of Mathematics

Faculty of Science

Kagoshima University

Kagoshima 890, Japan

E-mail: atsumi@sci.kagoshima-u.ac.jp 